\section{Stressing out over depression and anxiety}

\section{By Tim Fulmer, Senior Writer}

Two teams have shown that enhanced glucocorticoid signaling in the brain triggers aberrant behavior in mouse models of depression and anxiety. ${ }^{1,2}$ The groups are now using the mice to study the mechanisms underlying the effect and determine whether blocking glucocorticoid signaling can treat neuropsychiatric diseases.

All mammals respond to stress by releasing glucocorticoids from the adrenal glands. Although that stress mechanism is beneficial when it facilitates the fight or flight response, chronic activation of the glucocorticoid response can trigger multiple neuropsychiatric disorders in humans, including pathological anxiety, depression and addiction.

The challenge has been identifying the molecular mechanisms underlying chronic activation of the glucocorticoid-mediated stress response and determining whether blocking that mechanism could help treat those disorders. Moreover, because the glucocorticoid receptor (GCCR) is a transcription factor expressed in many different cell types, safely targeting it in neuropsychiatric disorders will require selectively hitting specific neurons in the brain.

To tackle those issues, two groups generated genetically altered mice and subjected them to environmental conditions predicted to trigger stress-mediated anxiety and depression in humans.

The French group, led by François Tronche and Jacques Barik, built on their prior work that showed selectively knocking out Gccr in postsynaptic, dopamine-sensing neurons decreased cocaine addiction in mice compared with what was seen in control mice. ${ }^{3}$ Dopamine signaling in the mesolimbic pathway mediates the addictive properties of cocaine and other drugs of abuse.

Tronche is director of research at the Pierre and Marie Curie University. Barik is a researcher at the university.

In the new work, Tronche and colleagues used the same mice to study whether selective Gccr knockout had a broader protective effect on mice undergoing chronic stress.

Indeed, in animals subjected to repeated social defeat, selective knockout of Gccr in postsynaptic, dopamine-sensing neurons led to normal social interactions, whereas wild-type animals showed significantly increased anxiety and social aversion $(p<0.01)$.

Moreover, the effects were localized to the postsynaptic neurons, as selective knockout of Gccr in presynaptic, dopamine-releasing neurons failed to protect the same animals from anxiety and social avoidance.
Subsequent mechanistic studies showed that protective Gccr knockout in postsynaptic neurons also decreased neurotransmission between preand postsynaptic dopamine neurons. That suggested pharmacological blockade of dopamine neuron activity might improve social interactions of wild-type mice following social defeat.

In wild-type mice undergoing repeated social defeat, injection of quinpirole, a generic small molecule that suppresses dopamine neuron activity, increased social interactions compared with injection of saline $(p<0.001)$.

In the second paper, a Japanese and American group hypothesized that environmental stresses in adolescence could be risk factors for depression and anxiety in adulthood.

To test that idea, a team led by Toshitaka Nabeshima and Akira Sawa generated transgenic mice expressing disrupted in schizophrenia 1 (DISC1), a gene implicated in the development of depression and schizophrenia, ${ }^{4}$ and subjected the adolescent animals to three weeks of isolation stress.

Nabeshima is professor of pharmacy at Meijo University. Sawa is professor of psychiatry and director of the Johns Hopkins Schizophrenia Center at The Johns Hopkins University School of Medicine.

The resulting animals showed significantly increased social deficits in multiple behavioral tests compared with wild-type controls $(p<0.01)$.

a Environmental stress

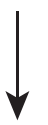

Release of glucocorticoid into blood

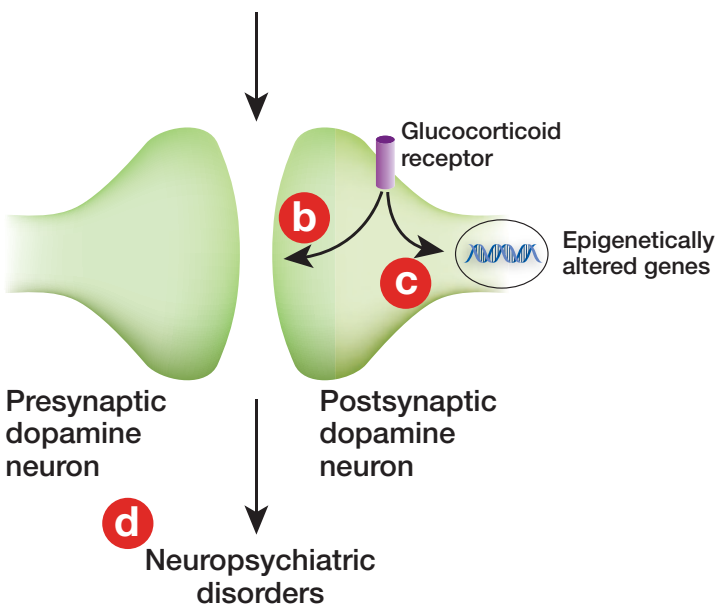

Figure 1. Dopamine stressed out over glucocorticoids. Environmental triggers induce the release of plasma glucocorticoids [a], which influence dopamine signaling in the brain by either directly altering dopamine neurotransmission between cells [b] or indirectly altering the epigenetic properties of genes involved in dopamine biosynthesis [c]. The resulting alterations lead to behavioral aberrations indicative of anxiety and depression [d]. 
The mice also had greater levels of dopamine and tyrosine hydroxylase (TH; TYH) in the frontal cortex. TH catalyzes the rate-limiting step in the synthesis of dopamine.

The increased dopamine levels were associated with greater plasma levels of the glucocorticoid corticosterone.

Based on the findings, the researchers next hypothesized that increased glucocorticoid levels might underlie the higher dopamine levels and thus indirectly drive the behavioral aberrations.

Indeed, in the same mice subjected to isolation stress, a small molecule GCCR antagonist normalized dopamine levels and improved social behaviors, whereas vehicle control had no effect.

Finally, genomic analysis of the mouse models revealed epigenetic alterations associated with isolation stress. Methylation of the promoter region of $\mathrm{TH}$ was significantly greater than that seen for the same gene in normal control animals $(p<0.01)$. Importantly, the same GCCR antagonist normalized DNA methylation levels of the gene.

In conclusion, the authors of the second paper wrote that their mice "may be a promising model for psychotic depression" and "could provide a good template not only for screening compounds with better efficacy and fewer side effects but also for prophylactic environmental readjustment."

Taken together, the two papers published back-to-back in Science suggest a mechanism whereby environmental triggers induce the release of plasma glucocorticoids, which then influence dopamine signaling in the brain by either directly altering dopamine neurotransmission between cells or indirectly altering the epigenetic properties of genes involved in dopamine biosynthesis. In either case, the resulting alterations lead to behavioral aberrations indicative of anxiety and depression (see Figure 1, "Dopamine stressed out over glucocorticoids").
The French group now plans to test strategies for selectively antagonizing GCCR in postsynaptic, dopamine-sensing neurons as well as approaches for decreasing the activity of dopamine neurons to reduce social aversion, co-corresponding author Tronche told SciBX.

The Japanese group plans to use its mice to determine whether the observed epigenetic alterations are limited to $\mathrm{TH}$ or also include other genes, said co-corresponding author Sawa.

The findings in both papers are not covered by IP and are unavailable for licensing.

Fulmer, T. SciBX 6(6); doi:10.1038/scibx.2013.130

Published online Feb. 14, 2013

\section{REFERENCES}

1. Barik, J. et al. Science; published online Jan. 18, 2013;

doi:10.1126/science. 1226767

Contact: François Tronche, Pierre and Marie Curie University,

Paris, France

e-mail: francois.tronche@upmc.fr

Contact: Jacques Barik, same affiliation as above

e-mail: jacques.barik@snv.jussieu.fr

2. Niwa, M. et al. Science; published online Jan. 18, 2013;

doi:10.1126/science.1226931

Contact: Akira Sawa, The Johns Hopkins University School of Medicine, Baltimore, Md.

e-mail: asawa1@jhmi.edu

Contact: Toshitaka Nabeshima, Meijo University, Nagoya, Japan e-mail: tnabeshi@meijo-u.ac.jp

3. Ambroggi, F. et al. Nat. Neurosci. 12, 247-249 (2009)

4. Brandon, N.J. et al. J. Neurosci. 29, 12768-12775 (2009)

COMPANIES AND INSTITUTIONS MENTIONED

The Johns Hopkins University School of Medicine,

Baltimore, Md.

Meijo University, Nagoya, Japan

Pierre and Marie Curie University, Paris, France 